\title{
Impaired Emotional Declarative Memory Following Unilateral Amygdala Damage
}

\author{
Ralph Adolphs, ${ }^{1}$ Daniel Tranel, and Natalie Denburg \\ Department of Neurology, Division of Cognitive Neuroscience, University of Iowa College of Medicine, Iowa City, Iowa 52242 USA
}

\begin{abstract}
Case studies of patients with bilateral amygdala damage and functional imaging studies of normal individuals have demonstrated that the amygdala plays a critical role in encoding emotionally arousing stimuli into long-term declarative memory. However, several issues remain poorly understood: the separate roles of left and right amygdala, the time course over which the amygdala participates in memory consolidation, and the type of knowledge structures it helps consolidate. We investigated these questions in eight subjects with unilateral amygdala damage, using several different measures. For comparison, our main task used stimuli identical to those used previously to investigate emotional declarative memory in patients with bilateral amygdala damage. Contrasts with both brain-damaged and normal control groups showed that subjects with left amygdala damage were impaired in their memory for emotional stimuli, despite entirely normal memory for neutral stimuli (because of a number of caveats, the findings from subjects with right amygdala damage were less clear). Follow-up experiments suggested that the normal facilitation of memory for emotional stimuli may develop over an extended time course (>30 min), consistent with prior findings, and that the specific impairment we report may depend in part on the lexical nature of the task used (written questionnaire). We stress the complex and temporally extended nature of memory consolidation and suggest that the amygdala may influence specific components of this process.
\end{abstract}

A large literature from studies in animals has demonstrated that the amygdala critically influences both the acquisition and the expression of emotional memories. Much of the evidence to support this claim comes from studies of motivated learning, using both appetitive as well as aversive stimuli. In these experiments, clear evidence has been obtained that the behavioral expression of emotionally motivated learning depends on the integrity of the amygdala during a narrow and highly specific window of time (McGaugh et al. 1992; Cahill and McGaugh 1998; Bianchin et al. 1999). Notably, the amygdala appears to play a critical role immediately after acquisition of the behavior and for some duration of time thereafter. These findings have been interpreted as evidence for the amygdala's role specifically in the consolidation processes of motivated learning that are influenced by emotional arousal, a finding also consistent with studies in humans (see below) (Cahill and McGaugh 1998; McGaugh 2000). Direct manipulations of neural activity in the amygdala with reversible pharmacological lesions have shown that the amygdala exerts its modulatory effect on secondary structures, such as the hippocampus, that are directly involved in memory consolidation (Packard et al. 1994; McGaugh et al. 1996; Packard and Teather 1998; Roozendaal et al. 1999).

Recently, the amygdala's role in emotional memory has been investigated also in humans. Five case studies of rare patients with selective bilateral amygdala damage provided evidence that the human amygdala is necessary for the en-

${ }^{1}$ Corresponding author.

E-MAIL ralph-adolphs@uiowa.edu; FAX (319) 356-4505. hanced memory normally seen with highly aversive, emotionally arousing stimuli (Babinsky et al. 1993; Cahill et al. 1995; Adolphs et al. 1997; Phelps et al. 1998; Hamann et al. 1999b). Functional imaging studies have shown that amygdala activation at the time that stimuli are first seen and encoded correlates with how well the same stimuli are remembered weeks later, but only in those cases where the stimuli are emotionally highly arousing (Cahill et al. 1996; Canli et al. 1998; Hamann et al. 1999a). There is thus good evidence that the human amygdala aids in the potentiation of memory traces for emotionally arousing stimuli during their acquisition and consolidation into long-term declarative memory.

Whereas animal studies have explicitly investigated unilateral effects of the amygdala on memory consolidation (e.g., Packard et al. 1994; Packard and Teather 1998), this has, in general, not been the case for studies in humans. In humans, one study examined the effect of unilateral amygdala damage on long-term recognition memory for emotional words (LaBar and Phelps 1998) and found impairments with either left or right damage. Functional imaging studies have provided inconsistent reports of the involvement of either the left (e.g., Lane et al. 1997) or right (e.g., Cahill et al. 1996) amygdala in encoding emotional stimuli. Most studies of emotional memory in subjects with bilateral amygdala damage (Cahill et al. 1995; Adolphs et al. 1997; Hamann et al. 1999b; but for additional tasks, see Phelps et al. 1998) used a task and set of stimuli developed by Reisberg and colleagues in normal subjects; however, this task has never been used in subjects with unilateral amygdala damage. Of particular interest would thus be an investiga-

LEARNING \& MEMORY 7:180-186 @ 2000 by Cold Spring Harbor Laboratory Press ISSN1072-0502/00 \$5.00

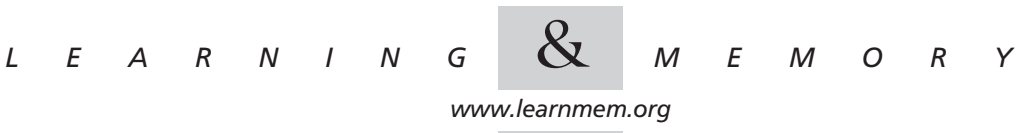


Table 1. Demographics and Background Neuropsychology of Subjects

\begin{tabular}{|c|c|c|c|c|c|c|}
\hline & Age & Education & PIQ & VIQ & Faces & BDI \\
\hline NC & $36 \pm 9$ & $12 \pm 0$ & & $98 \pm 10$ & & \\
\hline BDC & $45.5 \pm 12$ & $15.9 \pm 2$ & $110 \pm 15$ & $115 \pm 18$ & $47 \pm 5$ & $4 \pm 4$ \\
\hline L & $33 \pm 8$ & $14 \pm 2$ & $108 \pm 13$ & $94 \pm 17$ & $43 \pm 3$ & $5 \pm 7$ \\
\hline $\mathrm{R}$ & $30 \pm 7$ & $16 \pm 0$ & $116 \pm 23$ & $110 \pm 6$ & $43 \pm 0$ & $4 \pm 0$ \\
\hline
\end{tabular}

(NC/BDC) Normal and brain-damaged controls; (L/R) subjects with left or with right amygdala damage; (PIQ/VIQ) performance and verbal IQ from the WAIS-R or the WAIS-III; (Faces) raw score on the Benton facial recognition task (all in the normal range); (BDI) raw score on the Beck depression inventory (all not depressed).

tion of the effects of unilateral amygdala damage, using this same task that has been used to assess declarative emotional memory in subjects with bilateral amygdala damage, the topic of the present report.

We investigated emotional declarative memory in eight subjects with unilateral amygdala damage consequent to neurosurgical temporal lobectomy. To facilitate direct comparisons with prior studies, we used the identical stimuli and main task as those used in prior studies of subjects with bilateral amygdala damage (Cahill et al. 1995; Adolphs et al. 1997; Phelps et al. 1998; Hamann et al. 1999b). Subjects were shown 12 slides that told a story, and their memory for the stimuli was assessed with a standardized questionnaire $24 \mathrm{hr}$ later (Heuer and Reisberg 1990; Burke et al. 1992; Reisberg and Heuer 1992; Cahill et al. 1994; Cahill and McGaugh 1995). The story consisted of three distinct phases: Phase 1 (the first four slides) consisted of material that is normally judged to be relatively nonemotional, phase 2 (the middle four slides) contained emotionally arousing material (scenes of surgery at a hospital), and phase 3 (the last four slides) consisted again of relatively nonemotional material. Especially arousing is slide 7, in phase 2, which shows the surgically sutured legs of a carcrash victim; this slide has been reliably linked to the best memory performance in prior studies (Cahill and McGaugh 1995; Adolphs et al. 1997). Our findings were compared with two control groups: normal subjects with no brain damage and brain-damaged subjects with no damage to the amygdala.

In addition to the above new investigation, we explored further some of the factors that might contribute to the effects we report. We followed up our main experiment with two further investigations: (1) An additional visual recognition memory task was used to explore the possible dissociation of memory when assessed with a lexical questionnaire, as compared with visual recognition of the stimuli. (2) Administration of the same stimuli and task using a much shorter time interval (30 min) was used to explore the time course of memory consolidation.

\section{RESULTS}

Background neuropsychological and demographic data are given in Table 1 . In general, subject groups were similar with respect to these background measures, and subjects with amygdala damage were always approximately matched to at least one of the two control groups on each parameter. Whereas brain-damaged controls were, on average, older
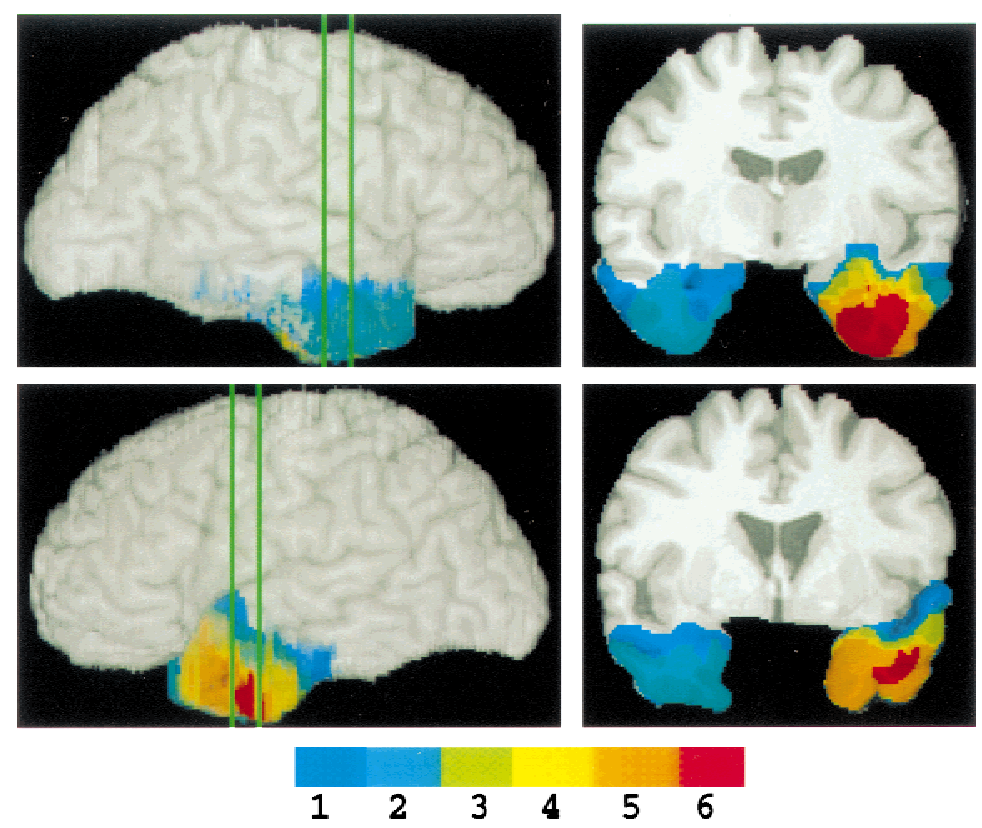

Figure 1 Summary of subjects' neuroanatomy. Subjects' lesions from MR scans were coregistered onto the corresponding MR sections of a single, normal reference brain. The joint overlaps of lesions from multiple subjects were encoded by color (scale showing total subject $N$ at bottom), and the MR sections of the normal brain with mapped lesions were then reconstructed in 3-D to obtain the whole brains shown in the figure (Frank et al. 1997). (Left) Left and right lateral views of the 3-D reconstructed brain with mapped lesions; (right) coronal sections through the middle and very anterior amygdala, corresponding to the planes indicated on the whole brains. Note that we follow the radiological convention of showing the right side of the brain on the left side of the coronal images.

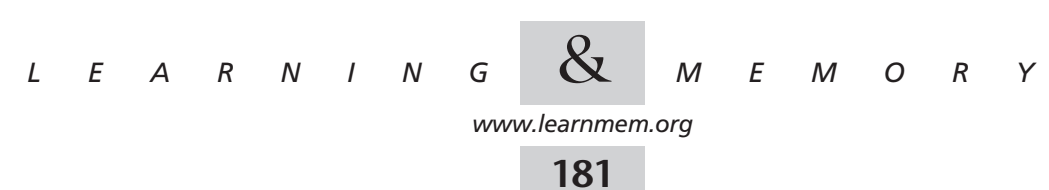




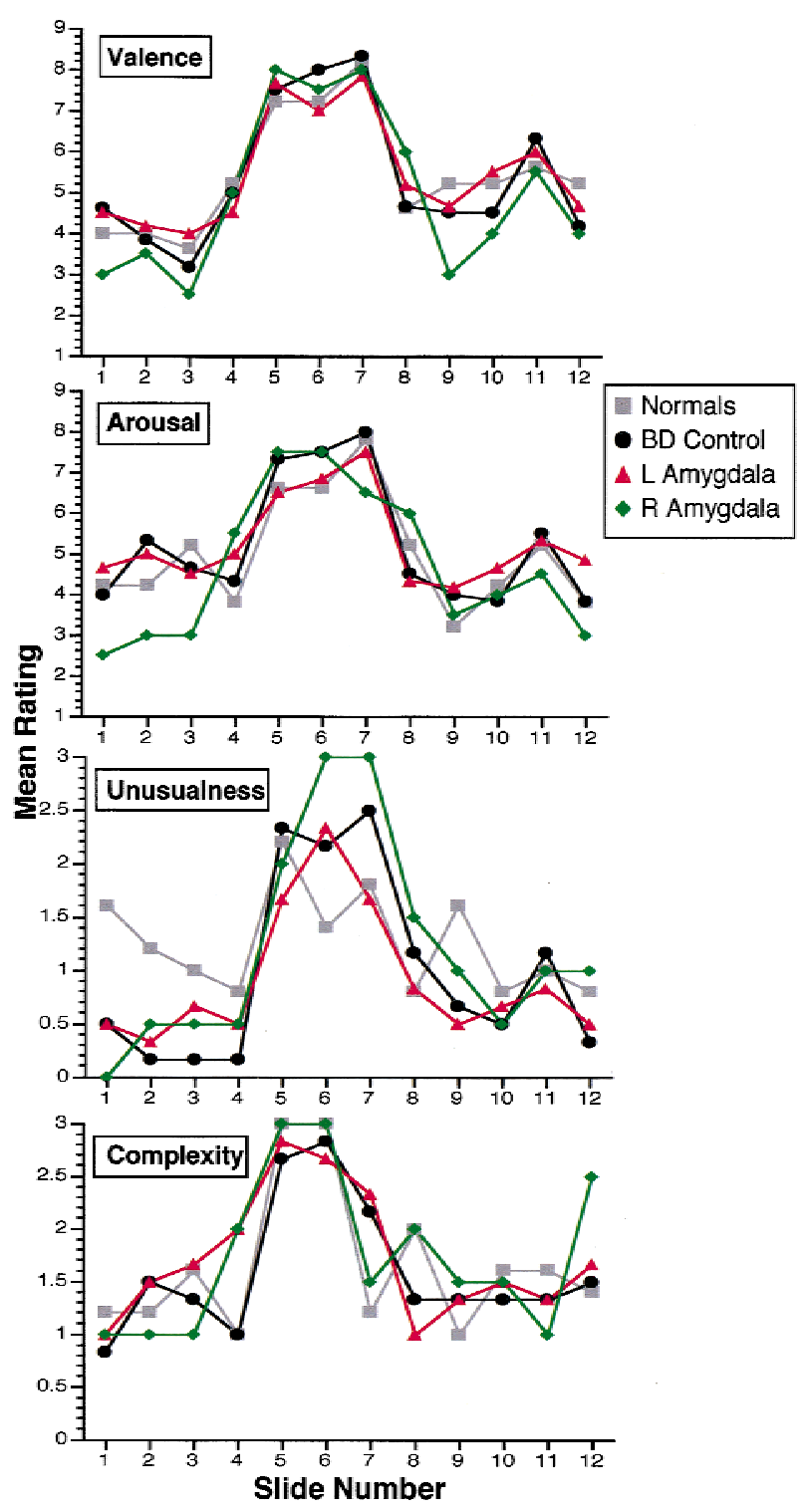

Figure 2 Ratings of the stimuli. Shown are ratings of valence (9point scale; 5 is neutral), arousal (9-point scale; 5 is neutral), perceived visual complexity (scale $0-3$ ), and perceived unusualness (scale 0-3). In all cases, subjects rated their own personal reaction or judgment. Data are from normal controls, brain-damaged controls, left amygdala damage, and right amygdala damage, as indicated. The $x$-axis ranks the stimuli in the order that they were

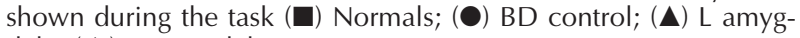
dala; ( $)$ R amygdala.

and had higher verbal IQ than subjects with amygdala damage, normal controls were of similar age and verbal IQ. Neuroanatomy of the subjects with amygdala damage is shown in Figure 1: All subjects had partial unilateral amygdala damage, as well as additional damage in anterior temporal lobe.

Ratings of the Stimuli

All subjects endorsed similar ratings for the stimuli on scales of valence, arousal, visual complexity, and unusualness, findings in keeping with previous reports that amygdala damage does not affect the way subjects judge or rate attributes of such stimuli (Cahill et al. 1995; Adolphs et al. 1997; Hamann et al. 1997). Figure 2 summarizes these data for each subject group, for each of the 12 stimuli. We conducted group (R, L, brain-damaged control, and normal control) by phase of story (1, nonemotional; 2 , highly emotional; 3, nonemotional) ANOVAs for each of the rating scales, using a subject's mean rating for the stimuli in a phase of the story to reveal the following effects of story phase on all scales: For valence, $F=30.5 ; P<0.0001$; for arousal, $F=17.2 ; \quad P<0.0001 ;$ for unusualness, $F=10$, $P<0.0005$; for complexity, $F=12 ; P<0.0001$. There were no significant effects of subject group nor any significant interactions of subject group with story phase on any rating scale.

\section{Memory Performance on 24-hr Delayed Questionnaire}

Brain-damaged controls and normal controls performed similarly on the questionnaire administered $24 \mathrm{hr}$ later and showed a pattern reported previously (Heuer and Reisberg 1990; Cahill and McGaugh 1995; Adolphs et al. 1997). In particular, control subjects remembered best information about a highly arousing slide that shows surgically reattached legs of a car-crash victim (slide no. 7), as reported in prior studies using the same stimuli (Cahill and McGaugh 1995; Adolphs et al. 1997). In contrast, subjects with left temporal lobectomy had impaired memory for slide 7 (Fig. 3). The impairment was statistically significant when comparing the memory performance for slide 7 of left temporal lobectomies versus normal controls $(P<0.005)$ but did not reach significance versus brain-damaged controls $(P=0.1$; Mann-Whitney $U$ tests). The finding is especially striking in view of the otherwise normal performance profile of left temporal lobectomy subjects on the task and in view of the

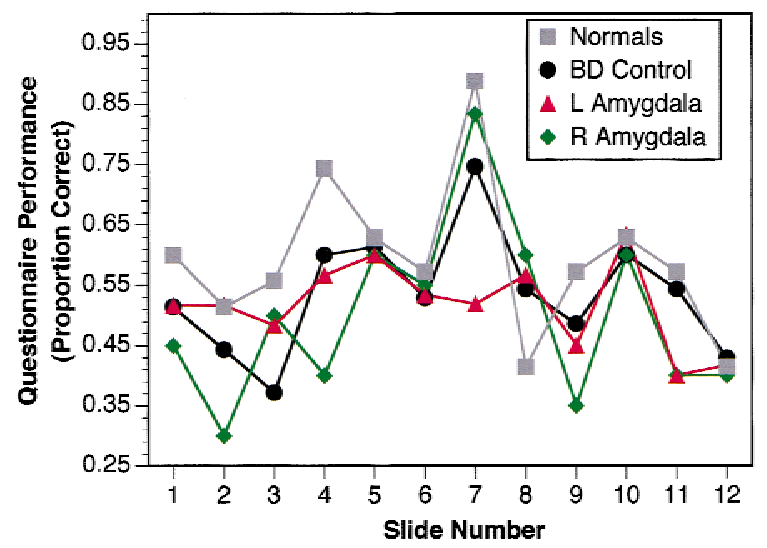

Figure 3 Memory performance on 24-hr delayed questionnaire. Proportion correct mean choices are shown for each subject group.

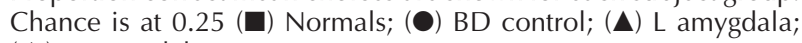
(৬) R amygdala.

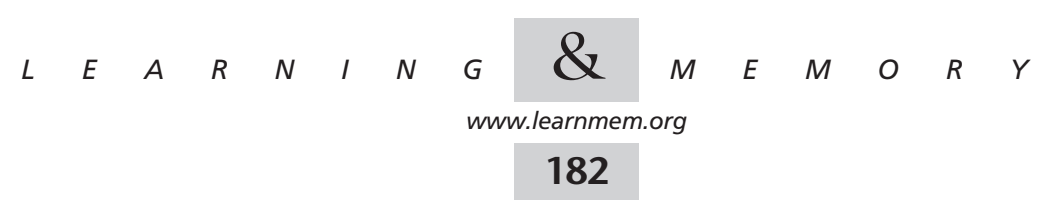


fact that subjects with right temporal lobectomy also performed normally. Whereas subjects with right temporal lobectomy, brain-damaged controls, and normal controls all showed the best memory for slide 7 , subjects with left temporal lobectomy did not (Fig. 3). This pattern of impairment is precisely what has been reported previously for subjects with bilateral amygdala damage (Adolphs et al. 1997).

\section{Visual Recognition Memory}

Visual recognition memory performances were similar for all subject groups (Fig. 4). In particular, recognition memory for slide 7 was the best of all the stimuli, for subjects with left temporal lobectomy as for controls. This finding is thus in contrast to the relative impairment for slide 7 when memory was assessed with the questionnaire.

\section{Effect of Short Delay}

A separate group of nine normal subjects participated in the memory questionnaire task after a 30-min delay, instead of a 24-hr delay. As Figure 5 shows, these subjects, unlike control subjects in the 24-hr delay condition, did not show a differential pattern of enhanced performance for the most arousing stimuli. In particular, there was no enhanced memory for slide 7: Normal controls given a 24-hr delay showed a disproportionately enhanced memory for this slide, whereas normal controls given a 30-min delay did not $(P<0.005$; Mann-Whitney $U$ test on memory for slide 7 normalized to memory over all stimuli for each subject).

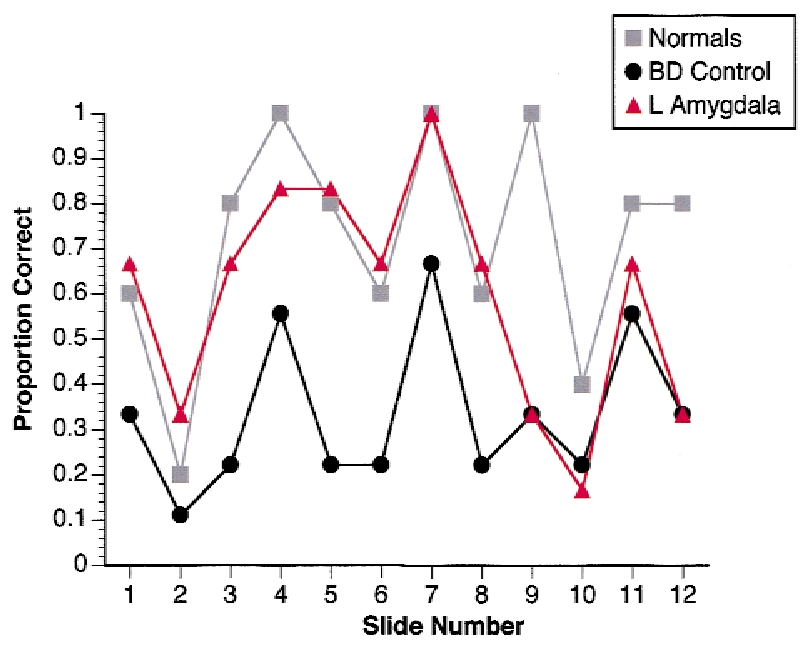

Figure 4 Memory performance on 24-hr delayed visual recognition. Proportion correct mean choices are shown for each subject group. Chance is at 0.25 . Data from the two subjects with right amygdala damage are not shown for clarity (both recognized slide 7 correctly and showed the same overall pattern as the other subject groups). Although all subject groups performed comparably on this task, this finding is subject to ceiling effects and may not reveal

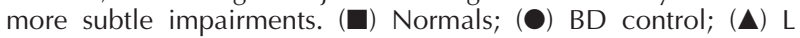
amygdala.

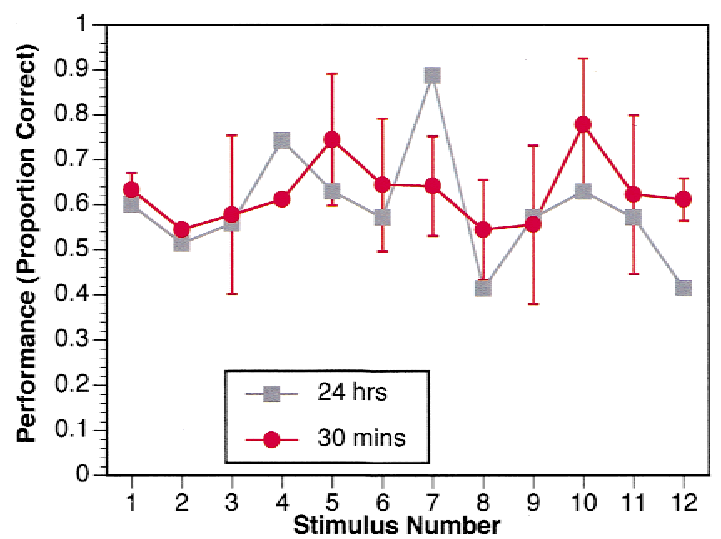

Figure 5 Memory performance on questionnaire after a 30-min delay. Data are from a separate group of nine normal controls and demonstrate that the enhanced memory for emotionally arousing material is a pattern that requires longer than $30 \mathrm{~min}$ to appear. For comparison, the data from normal subjects with the 24-hr delay are shown as well. (ם) $24 \mathrm{hr}$; (@) $30 \mathrm{~min}$.

\section{DISCUSSION}

\section{Summary of Findings}

1. In the task we used, emotionally arousing stimuli are normally remembered better than neutral stimuli, but this effect requires a delay longer than 30 min to appear (Figs. 3 and 5).

2. Subjects with unilateral amygdala damage were impaired in their long-term (24-hr) declarative memory for emotional stimuli, when memory was assessed with a standard multiple-choice questionnaire (Fig. 3). This finding was clear only for subjects with left amygdala damage.

3. There was no clear evidence that two subjects with right unilateral amygdala damage were impaired on the same task (Fig. 3).

4. There was no evidence that any subject group was impaired in visual recognition memory, and all showed a similar pattern of performances (Fig. 4); however, these findings are subject to ceiling effects.

5. All subject groups rated the stimuli similarly in terms of valence, arousal, complexity, and unusualness (Fig. 2).

Thus, left unilateral amygdala damage impairs longterm declarative memory for emotionally arousing stimuli despite normal emotional ratings of the stimuli and despite apparently normal visual recognition memory. Taken together with findings from other studies, these data support the hypothesis that the left amygdala is critical to consolidate declarative memory for emotionally arousing stimuli. Furthermore, the role of the left amygdala may be especially important to restructure memory for the stimuli so as to incorporate both lexical and visual knowledge. The role of the right amygdala remains to be explored further; our small sample size does not permit any definite conclusions at this point.

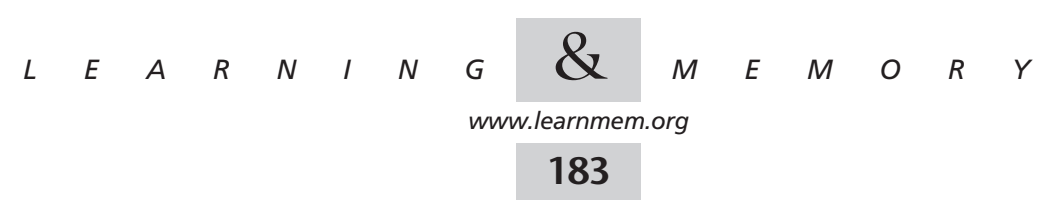




\section{Caveats}

Our interpretations are subject to some caveats and leave several alternate possibilities open. Most importantly, the findings cannot unambiguously implicate the amygdala as the sole structure responsible for the impairments reported, because although all subjects had partial unilateral amygdala damage, all had additional damage to entorhinal cortex, white matter, and neocortex in anterior temporal lobe. Any or all of these regions could contribute to emotional memory, and future studies with selective lesions, or functional imaging studies in normals, will be necessary to distinguish their relative importance. Given prior findings that do demonstrate the amygdala's role in emotional declarative memory, we nonetheless find it very likely that amygdala damage explains most, if not all, of the findings reported here.

Likewise, we also cannot make a strong claim regarding the laterality of amygdala damage. Although we found evidence to support the claim that left amygdala damage impairs emotional memory, it would be premature to conclude that right amygdala damage results in no such impairment. Our sample sizes were not matched (six left, two right), and the small number of subjects with right amygdala damage does not permit any definitive conclusions at this point. Nonetheless, our findings are consistent with studies that showed a lateralized activation in the left amygdala when subjects consciously experienced emotions (Lane et al. 1997; Schneider et al. 1997; Morris et al. 1998). Encoding emotional stimuli into memory has been reported to disproportionately activate the left amygdala in some studies (Taylor et al. 1998; but see Cahill et al. 1996), and there also are reports of sparing of visual memory in subjects with right amygdala pathology (Loring et al. 1989; Cohen et al. 1990), depending on the precise task used. On the other hand, the most relevant study, which investigated emotional memory for words following unilateral amygdala damage (LaBar and Phelps 1998), found equal impairments for both right- and left-damaged subjects, although their left-damage subjects had a lower baseline memory performance. Taken together, the findings support a clear role for the left amygdala in emotional memory; although the right amygdala is also very likely to play a role, this may be somewhat less critical or less easy to detect with our particular stimuli and task.

A final caveat concerns the nature of the stimuli. We used the particular stimuli and task because these constitute an established protocol that has been used in several prior studies of normal subjects and of subjects with bilateral amygdala damage. However, the design of the task leaves open several questions. Notably, the data from the visual recognition memory task (Fig. 4) are clearly subject to ceiling effects and thus make this task insensitive to possible impairments; the conclusion that subjects with amygdala damage are normal on this task needs to be interpreted with caution. Furthermore, there are questions regarding the primary stimulus factors responsible for the normal enhance- ment of memory seen. In particular, as can be seen from Figure 2, the most unpleasant stimuli are also the most emotionally arousing and unusual. All findings from this task, taken in isolation, are thus consistent with the possibility of an effect of valence, arousal, unusualness, or any combination of these different factors. However, other studies have argued that emotional arousal is likely to be the primary factor responsible for the enhanced memory observed (Bradley et al. 1992; Cahill et al. 1994; Hamann et al. 1999a); studies in patients with unilateral amygdala damage have shown explicitly that emotional valence is not sufficient, by itself, to account for the impairments in emotional memory found in such patients (Phelps et al. 1997). Ongoing studies in our laboratory are attempting to dissect the contributions of all these different factors to memory and their possible dependency on the amygdala.

\section{The Amygdala in Memory Consolidation}

This study provides several related findings: Unilateral damage to the amygdala impairs emotional memory, memory for emotional stimuli is normally enhanced only after a substantial temporal delay, and the particular impairment we reported may be disproportionately severe when assessing memory using lexical instruments (a questionnaire) rather than visual recognition. The first finding is consistent with the idea that the human amygdala aids in the consolidation of long-term declarative memory for emotional events, a conclusion already supported by studies in subjects with bilateral amygdala damage, but extends it to show that unilateral amygdala damage may be sufficient to result in impairment. The other findings provide some additional details.

First, the enhanced memory for emotional stimuli appears to emerge over many hours, possibly overnight, making it plausible that the amygdala participates in memory consolidation over that time period. This idea would be consistent with the temporally extended role of the amygdala in memory consolidation in animal studies (McGaugh et al. 1996; Bianchin et al. 1999) and also with the finding that the human amygdala is especially active during REM sleep (Maquet et al. 1996), a state that may play a key role in memory consolidation (Stickgold 1999). One plausible component of this consolidation would be the disproportionate forgetting of neutral material together with preserved or enhanced memory for emotional material.

This issue has been investigated in a number of studies in normal individuals, which have found that memory for arousing verbal material increases over time (perhaps up to a week or more), whereas memory for neutral verbal material decreases with time (Kleinsmith and Kaplan 1963); furthermore, it has been demonstrated that this effect on memory can result from arousal alone, when arousal is manipulated independently of the stimuli (Berlyne et al. 1965; see also Cahill et al. 1994). A prior study (LaBar and Phelps 1998) argued that the amygdala may participate in this dif-

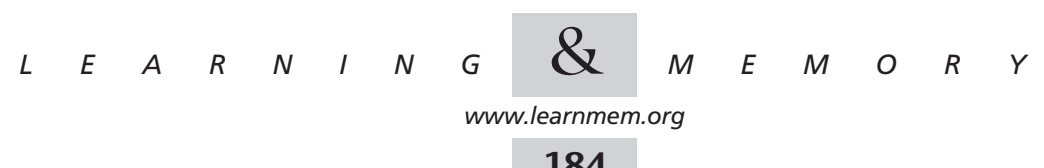


ferential memory for material over time as a function of emotional arousal. Thus, normal subjects might, in part, transform their performances after $30 \mathrm{~min}$ into those after $24 \mathrm{hr}$ (Fig. 5) by forgetting some of the more neutral material but not emotional material. Our finding is thus consistent with the findings by LaBar and Phelps (1998) (see also Phelps et al. 1998), as well as with older studies that have proposed the same mechanism (Kleinsmith and Kaplan 1963). However, as also pointed out by these other studies on memory for verbal material (Kleinsmith and Kaplan 1963; LaBar and Phelps 1998), forgetting is unlikely to be the only mechanism by which to explain our data, because there are some slides that appear to show an increase in memory score over time. In general, a comparison of the 30-min and the 24-hr memory performances (Fig. 5) suggests a differential sharpening of the memory profile for the stimuli: Some stimuli are remembered less well at $24 \mathrm{hr}$, whereas others are remembered better. It thus appears likely that both mechanisms that involve forgetting, as well as mechanisms that involve enhancement, are operating over time, a complex issue that will require further investigation to tease apart possible subcomponents of this process that rely on the amygdala.

Second, the findings point toward a possible role of the left amygdala in transforming visual information about a stimulus image into an elaborated memory structure that includes information accessible to, or encoded in, language. The left amygdala thus may be especially important in facilitating the consolidation of emotionally relevant visual information, together with lexical information from the narrative and lexical information generated internally by the subject. Such lexical knowledge structures may be generated during normal memory consolidation to elaborate a coherent, long-term schema, in essence, a story encoded in language that links visual, emotional, and narrative information acquired at the time the stimuli were shown. Although our study, because of the small sample size, did not reveal any specific impairments in subjects with right amygdala damage, we nonetheless consider it likely that the right amygdala will also participate in the above processes, probably in concert with the left amygdala. The precise roles of the left and the right amygdala in such a complex set of consolidation processes will require further study in which very specific aspects of memory function are assessed, a goal that will require the design of more specific tasks.

\section{MATERIALS AND METHODS}

\section{Subjects}

Participants were eight subjects with unilateral amygdala damage consequent to neurosurgical temporal lobectomy for the treatment of epilepsy, nine brain-damaged subjects with no damage to anterior temporal lobe, and seven normal controls (mean age $36 \pm 8.5$ ) whose data have been published previously (Adolphs et al. 1997). An additional, separate group of nine normal controls (mean age
$35 \pm 9$ ) were the sole participants in a follow-up task in which memory was assessed after a short delay (see below).

All subjects with unilateral amygdala damage (six left: five male, one female; two right: one male, one female) were fully right-handed (+100 on the Oldfield-Geschwind questionnaire) and had damage that included temporal pole, anterior entorhinal cortex, and amygdala (Fig. 1). All were entirely seizure free except one of the subjects with right temporal lobectomy, who very rarely experienced mild seizures ( $<1 /$ year). Some of the temporal lobectomy subjects were taking low doses of antiseizure medication; however, post hoc investigations revealed that this did not contribute to impaired performance on the experimental tasks we report (some of the most impaired subjects were taking no medications whatsoever, whereas some who performed entirely normally were taking medication). Details on demographics, neuropsychology, and neuroanatomy for all subjects are given in Table 1 .

\section{Experimental Task}

To study the modulation of declarative memory by emotion, we used a task that has been administered to normal human subjects in several studies, with reliable findings (Heuer and Reisberg 1990; Burke et al. 1992; Reisberg and Heuer 1992; Cahill and McGaugh 1995). We (Adolphs et al. 1997) and others (Cahill et al. 1995; Hamann et al. 1999b) have used previously the identical task with rare subjects who have bilateral amygdala damage. The task consists of a set of 12 slides that are shown in sequence, accompanied by a narrative. Together, the slides and narrative tell a story about a boy and his mother who visit the father at the hospital. The story can be divided into three phases: Phase 1 (the first four slides) consists of material that is normally judged to be relatively nonemotional (the boy and mother are on their way to the hospital), phase 2 (the middle four slides) contains emotionally arousing material (scenes of surgery at the hospital), and phase 3 (the last four slides) consists again of relatively nonemotional material (the mother leaves the hospital to make a phone call and goes home). Especially arousing is slide 7 , in phase 2, which shows the surgically sutured legs of a car-crash victim; this slide has been reliably linked to the best memory performance in prior studies (Cahill and McGaugh 1995; Adolphs et al. 1997). The verbatim narrative that accompanied the slides is given in a prior publication (Adolphs et al. 1997).

Subjects were told to pay close attention to the stimuli, but subjects were not told, and did not expect, that this was a test of memory. Slides were shown projected on a wall in a darkened room (size of images $=1 \times 1.5 \mathrm{~m}$ ), and the narrative was spoken by the experimenter. Stimuli were shown for $20 \mathrm{sec}$, with no interstimulus interval. Subjects returned to our laboratory $24 \mathrm{hr}$ later, in each case after a night's sleep, and participated in the following tasks, in the fixed order given below.

1. Questionnaire: Twenty-four-hour delayed memory for the stimuli was assessed in detail using an established multiplechoice questionnaire (Heuer and Reisberg 1990), as in all prior studies that have used the same stimuli. The questionnaire asked 9-10 questions about each slide. All subjects participated in this task.

2. Visual recognition memory: Subjects were shown the original stimuli, together with three computer-manipulated foils in which some of the central characters or objects in the original image had been altered. Each stimulus was shown together with its three foils in a four-alternative forced-choice task; subjects had to point to the correct image. Five of the normal controls,

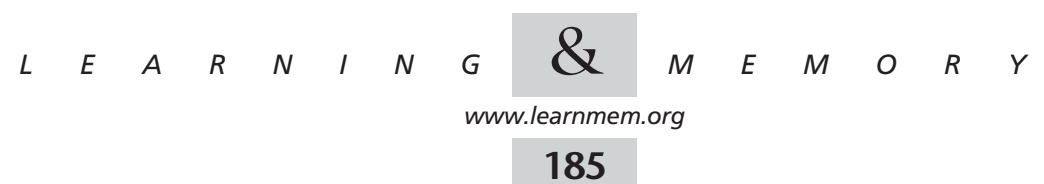


seven of the brain-damaged controls, and all eight subjects with temporal lobectomy participated in this task.

3. Ratings of the stimuli: Subjects then saw the stimuli again and rated them on Likert scales with respect to the following attributes: valence (degree of pleasantness from 1 to 9), arousal (intensity of emotional arousal from 1 to 9), visual complexity $(0-3)$, and unusualness (0-3). It was stressed that subjects were to rate their own personal response and judgment of the stimuli, not how they thought people would rate the stimuli in general. Five of the normal controls, six of the brain-damaged controls, and all subjects with temporal lobectomy participated in this task.

In addition to the above tasks, an additional nine normal subjects (separate from the seven who participated in the above tasks) were shown the same stimuli but filled out the same memory questionnaire (task 1 above) $30 \mathrm{~min}$ later, instead of $24 \mathrm{hr}$ later. They participated in no other tasks than the memory questionnaire, and their results are presented separately in Results.

\section{ACKNOWLEDGMENTS}

We thank Jeremy Nath and Kristofer Kinsey for help with testing subjects and Denise Krutzfeldt for help in scheduling their visits. Supported by a FIRST Award from NIMH to R.A.

The publication costs of this article were defrayed in part by payment of page charges. This article must therefore be hereby marked "advertisement" in accordance with 18 USC section 1734 solely to indicate this fact.

\section{REFERENCES}

Adolphs, R., L. Cahill, R. Schul, and R. Babinsky. 1997. Impaired declarative memory for emotional material following bilateral amygdala damage in humans. Learn. E Mem. 4: 291-300.

Babinsky, R., P. Calabrese, H.F. Durwen, H.J. Markowitsch, D. Brechtelsbauer. 1993. The possible contribution of the amygdala to memory. Behav. Neurol. 6: 167-170.

Berlyne, D.E., D.M. Borsa, M.A. Craw, R.S. Gelman, and E.E. Mandell. 1965. Effects of stimulus complexity and induced arousal on paired-associate learning. J. Verbal Learn. Verbal Behav. 4: 291-299.

Bianchin, M., T. Mello e Souza, J.H. Medina, and I. Izquierdo. 1999. The amygdala is involved in the modulation of long-term memory, but not in working or short-term memory. Neurobiol. Learn. Mem. 71: $127-131$

Bradley, M.M., M.K. Greenwald, M.C. Petry, and P.J. Lang. 1992 Remembering pictures: Pleasure and arousal in memory. J. Exp. Psychol. Learn. Mem. \& Cog. 18: 379-390.

Burke, A., F. Heuer, and D. Reisberg. 1992. Remembering emotional events. Mem. Cognit. 20: 277-290.

Cahill, L. and J.L. McGaugh. 1995. A novel demonstration of enhanced memory associated with emotional arousal. Consciousness Cognit. 4: $410-421$.

. 1998. Mechanisms of emotional arousal and lasting declarative memory. Trends Neurosci. 21: 294-299.

Cahill, L., B. Prins, M. Weber, J.L. McGaugh. 1994. Beta-adrenergic activation and memory for emotional events. Nature 371: 702-704.

Cahill, L., R. Babinsky, H.J. Markowitsch. and J.L. McGaugh. 1995. The amygdala and emotional memory. Nature 377: 295-296.

Cahill, L., R.J. Haier, J. Fallon, M.T. Alkire, C. Tang et al. 1996. Amygdala activity at encoding correlated with long-term, free recall of emotional information. Proc. Natl. Acad. Sci. 93: 8016-8021.

Canli, T., Z. Zhao, J. Desmond, G. Glover, and J.D.E. Gabrieli. 1998. Amygdala activation at encoding correlates with long-term recognition memory for emotional pictures: An fMRI study. Soc. Neurosci. Abstr. 24: 935

Cohen, N.J., G.L. Holmes, R. Campbell, and J.R. Smith. 1990. Memory performance following unilateral electrical stimulation of the hippocampus of a child with right temporal lobe epilepsy. J. Epilepsy 3: 115-122.
Frank, R.J., H. Damasio, and T.J. Grabowski. 1997. Brainvox: An interactive, multi-modal visualization and analysis system for neuroanatomical imaging. NeuroImage 5: 13-30.

Hamann, S.B., L. Cahill, and L.R. Squire. 1997. Emotional perception and memory in amnesia. Neuropsychology 11: 104-113.

Hamann, S.B., T.D. Ely, S.T. Grafton, and C.D. Kilts. 1999a. Amygdala activity related to enhanced memory for pleasant and aversive stimuli. Nat. Neurosci. 2: 289-293.

Hamann, S.B., G.P. Lee, and R. Adolphs. 1999b. Impaired declarative emotional memory but intact emotional responses following human bilateral amygdalotomy. Soci. Neurosci. Abstr. 25: 99.

Heuer, F. and D. Reisberg. 1990. Vivid memories of emotional events: The accuracy of remembered minutiae. Mem. Cognit. 18: 496-506.

Kleinsmith, L.J. and S. Kaplan. 1963. Paired-associate learning as a function of arousal and interpolated interval. J. Exp. Psychol. 67: 124-126.

LaBar, K.S. and E.A. Phelps. 1998. Arousal-mediated memory consolidation: Role of the medial temporal lobe in humans. Psychol. Sci. 9: 490-494

Lane, R.D., E.M. Reiman, M.M. Bradley, P.J. Lang, G.L. Ahern et al. 1997. Neuroanatomical correlates of pleasant and unpleasant emotion. Neuropsychologia 35: 1437-1444.

Loring, D.W., G.P. Lee, R.C. Martin, and K.J. Meador. 1989. Verbal and visual memory index discrepancies from the Wechsler Memory Scale-Revised: Cautions in interpretation. Psychol. Assess. 1: 198-202.

Maquet, P., J.-M. Peters, J. Aerts, G. Delfiore, C. Degueldre et al. 1996. Functional neuroanatomy of human rapid-eye-movement sleep and dreaming. Nature 383: 163-166.

McGaugh, J.L. 2000. Memory-A century of consolidation. Science 287: 248-251.

McGaugh, J.L., I.B. Introini-Collison, L. Cahill, M. Kim, and K.C. Liang. 1992. Involvement of the amygdala in neuromodulatory influences on memory storage. In The amygdala: Neurobiological aspects of emotion, memory, and mental dysfunction (ed. J.P. Aggleton) Wiley-Liss, New York, NY.

McGaugh, J.L., L. Cahill, and B. Roozendaal. 1996. Involvement of the amygdala in memory storage: Interaction with other brain systems. Proc. Natl. Acad. Sci. 93: 13508-13514.

Morris, J.S., A. Oehman, and R.J. Dolan. 1998. Conscious and unconscious emotional learning in the human amygdala. Nature 393: 467-470.

Packard, M.G. and L.A. Teather. 1998. Amygdala modulation of multiple memory systems: Hippocampus and caudate-putamen. Neurobiol. Learn. Mem. 69: 163-203.

Packard, M.G., L. Cahill, and J.L. McGaugh. 1994. Amygdala modulation of hippocampal-dependent and caudate nucleus-dependent memory processes. Proc. Natl. Acad. Sci. 91: 8477-8481.

Phelps, E.A., K. LaBar, and D.D. Spencer. 1997. Memory for emotional words following unilateral temporal lobectomy. Brain Cognit. 35: 85-109.

Phelps, E.A., K. LaBar, A.K. Anderson. K.J. O'Connor, R.K. Fulbright, and D. Spencer. 1998. Specifying the contributions of the human amygdala to emotional memory: A case study. Neurocase 4: 527-540.

Reisberg, D. and F. Heuer. 1992. Remembering the details of emotional events. In Affect and accuracy in recall: Studies of "flashbulb" memories (eds. E. Winograd and U. Neisser), pp. 163-190. Harvard University Press, Cambridge, MA.

Roozendaal, B., B.T. Nguyen, A.E. Power, and J.L. McGaugh. 1999. Basolateral amygdala noradrenergic influence enables enhancement of memory consolidation induced by hippocampal glucocorticoid receptor activation. Proc. Natl. Acad. Sci. 96: 11642-11647.

Schneider, F., W. Grodd, U. Weiss, U. Klose, K.R.M. et al. 1997. Functional MRI reveals left amygdala activation during emotion. Psychiat. Res. 76: 75-82.

Stickgold, R. 1999. Sleep: Off-line memory reprocessing. Trends Cognit Sci. 2: 484-492.

Taylor, S.F., I. Liberzon, L.M. Fig, L.R. Decker, S. Minoshima et al. 1998. The effect of emotional content on visual recognition memory: A PET activation study. Neuroimage 8: 188-197.

Received January 28, 2000; accepted in revised form March 27, 2000.

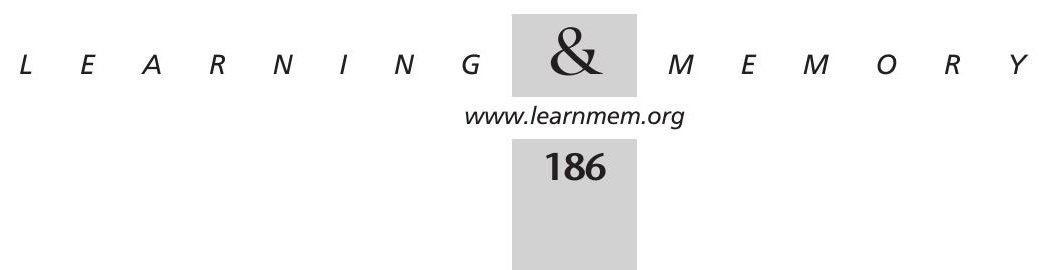




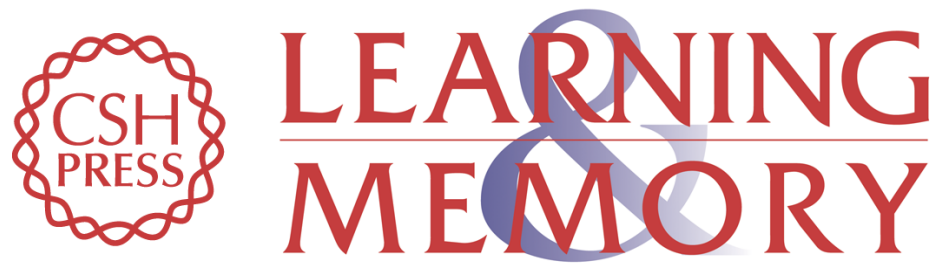

\section{Impaired Emotional Declarative Memory Following Unilateral Amygdala Damage}

Ralph Adolphs, Daniel Tranel and Natalie Denburg

Learn. Mem. 2000, 7:

Access the most recent version at doi:10.1101//m.7.3.180

References This article cites 31 articles, 6 of which can be accessed free at: http://learnmem.cshlp.org/content/7/3/180.full.html\#ref-list-1

License

Email Alerting Receive free email alerts when new articles cite this article - sign up in the box at the Service top right corner of the article or click here. 